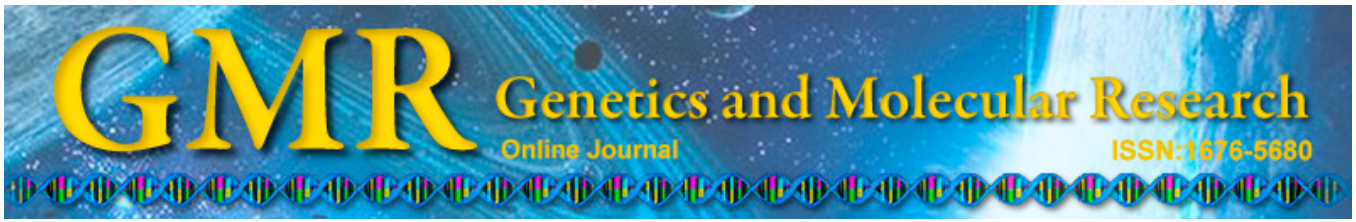

\title{
Adjuvant chemotherapy may improve survival of patients with luminal $A$ breast cancer and positive lymph nodes
}

\author{
Y. Han, Q. Li, B.H. Xu, P. Zhang, P. Yuan, J.Y. Wang, F. Ma, \\ R.G. Cai and Y. Fan \\ Department of Medical Oncology, Cancer Hospital, \\ Chinese Academy of Medical Sciences, Peking Union Medical College, \\ Chaoyang District, Beijing, China \\ Corresponding author: Q. Li \\ E-mail: QingLicn@163.com
}

Genet. Mol. Res. 14 (3): 8563-8573 (2015)

Received July 18, 2014

Accepted April 6, 2015

Published July 31, 2015

DOI http://dx.doi.org/10.4238/2015.July.31.4

\begin{abstract}
The study examined the clinicopathological characteristics and treatment options in patients with luminal A breast cancer. This retrospective cohort included 1580 patients with luminal A breast cancer treated between January 2005 and June 2007. Patients were divided into four subgroups according to lymph node status. Prognostic factors and 5-year overall survival (OS) and disease-free survival (DFS) of patients were analyzed. The median duration of follow-up was 67 months. Multivariate Cox-regression analysis revealed that patients in the LN2 and LN3 subgroups had a higher risk of recurrence and death than patients in the LN0 subgroup (LN2: HR $=2.2$ for DFS and HR $=2.1$ for OS; LN3: HR $=4.7$ for DFS and $\mathrm{HR}=4.7$ for OS). In the LN2 subgroup, there was a trend towards reduced risk of recurrence and death for patients receiving adjuvant chemotherapy plus endocrine therapy, although this difference did not reach statistical significance. In the LN0 and LN1 subgroups, there was a trend towards an increased risk of death in patients receiving chemotherapy. Although lymph node status
\end{abstract}


remains one of the most important independent prognostic predictors for luminal A breast cancer, in patients with 0-3 positive lymph nodes endocrine therapy can be considered sufficient. However, patients with $\geq 4$ positive lymph nodes, and especially in those with $\geq 10$, should receive chemotherapy.

Key words: Luminal A breast cancer; Lymph node status;

Overall survival; Adjuvant chemotherapy; Disease-free survival

\section{INTRODUCTION}

Breast cancer is a highly heterogeneous malignancy considering its morphological spectrum, clinical presentation, and response to therapy (Di Cosimo and Baselga, 2010). Based on gene expression profiling using cDNA microarrays, a molecular taxonomy-based classification has been proposed to divide breast cancer into luminal A, luminal B, basal-like, and human epidermal growth factor receptor 2 (HER2) subtypes, which exhibit distinct differences in prognosis and response to therapy (van't Veer et al., 2002; Sotiriou et al., 2003). The definition of luminal A disease includes cancers that are estrogen receptor (ER) and/or progesterone receptor $(\mathrm{PgR})$ positive and HER2 negative with a low Ki-67 proliferation index (<14\%) (Nielsen et al., 2004; Hugh et al., 2009; Cheang et al., 2009; Blows et al., 2010).

These subtypes have different epidemiological risk factors (Millikan et al., 2008; Phipps et al., 2011b), diverse natural histories (Liedtke et al., 2008; Dignam et al., 2009; Phipps et al., 2011a), and respond differently to systemic and local therapies (Nguyen et al., 2008; Albain et al., 2010; Wo et al., 2010; Tang et al., 2011; Aebi et al., 2011). Previous studies have shown no benefit of chemotherapy in the subset with high levels of endocrine receptors, negative HER2, and low Ki-67 (Colleoni et al., 2010), corresponding to the surrogate definition of luminal A disease (Cheang et al., 2009). Retrospective analysis of the National Surgical Adjuvant Breast and Bowel Project B-20 trial in patients with nodenegative disease found no benefit of adding chemotherapy to tamoxifen except in patients with the highest levels of recurrence score as measured by the OncotypeDX test (Paik et al., 2006). Similarly, among postmenopausal women with node-positive disease, SWOG 8814 (Albain et al., 2010) demonstrated no benefit of the addition of cyclophosphamide, doxorubicin, and fluorouracil chemotherapy to tamoxifen for those with high ER levels and negative HER2, and specifically for those with low or intermediate recurrence score, which would include virtually all patients with luminal A disease. Due to the good prognosis and poor response to chemotherapy of luminal A breast cancer, the St. Gallen Panel has advised that endocrine therapy alone should be administered in patients with clinicopathologically classified luminal A disease, even if a small proportion of patients should also receive cytotoxic drugs (Goldhirsch et al., 2011).

In our clinical experience, we have encountered many patients who were classified as having luminal A breast cancer with lymph node metastasis, and it would be important to know if these patients should undergo chemotherapy. This would thus avoid overtreatment and undertreatment for luminal A disease. To address these aspects, we conducted a retrospective study in luminal A breast cancer patients who underwent treatment and long-term followup at our institution. 


\section{MATERIAL AND METHODS}

\section{Patients and data collection}

Data was collected on patients referred for surgery to the Cancer Institute and Hospital, Chinese Academy of Medical Sciences, between January 2005 and June 2007. None of the patients had distant metastases. Data on medical history, concurrent diseases, surgery, pathological evaluations, and results of staging procedures were obtained. Pathological assessment included evaluation of the size of the primary tumor, histological type, and lymph node status, including sentinel node biopsy (Viale et al., 1999) when applicable. Tumor grade was evaluated according to the procedure described by Elston and Ellis (1991), and peritumoral vascular invasion was assessed as described by Rosen and Oberman (1993). ER and PgR status, Ki-67 labeling index, and HER2 overexpression (routinely carried out since 1999) were evaluated, and immunohistochemistry was performed as previously reported (Viale et al., 2008b). Tumor stage was determined according to criteria established by the 7th edition of the American Joint Committee on Cancer in the Cancer Staging Manual (Nguyen et al., 2008).

The Ki-67 labeling index is important in distinguishing between 'luminal A' and 'luminal B (HER2-)' breast cancer subtypes. Our study was a retrospective analysis, as we enrolled patients who underwent surgery between January 2005 and June 2007. During that period, our laboratory did not routinely determine the Ki-67 labeling index for pathological diagnosis of breast cancer. We chose, instead, to examine histological grade, as suggested by the St. Gallen International Breast Cancer Conference Expert Panel. The Nottingham modification of the Scarff-Bloom-Richardson histological grading is recognized as a treatment-related indicator, particularly in patients selected with grade 1 or 2 tumors who do not need adjuvant chemotherapy (Goldhirsch et al., 2009). Therefore, we selected our patients with grade I or II tumors, or with a Ki-67 labeling index $<15 \%$, and who were ER+ and/or PgR+ and HER2-.

\section{Treatments}

All patients underwent appropriate local treatment (breast-conserving surgery or total mastectomy) and axillary sentinel lymph node biopsy or complete axillary dissection. Postoperative breast irradiation was proposed for all patients who underwent breast-conserving surgery. Systemic adjuvant therapy was recommended according to the St. Gallen treatment guidelines (Nguyen et al., 2008; Goldhirsch et al., 1995, 2009). Adjuvant endocrine therapy was indicated, and chemotherapy was administered to 1206 patients. Anthracycline-containing chemotherapy, such as 4 or 6 courses of adriamycin and cyclophosphamide, was considered as the preferred option in all patients.

\section{Statistical analysis}

The primary endpoints of this study were disease-free survival (DFS) and overall survival (OS). The DFS period was defined as the interval from the date of diagnosis to the date when disease recurrence, either loco-regional or distant metastasis, was first observed, or the date of last follow-up without any evidence of recurrence. OS was calculated from the date of diagnosis of the primary breast cancer to the date of death or last follow-up.

To compare the clinicopathological characteristics of patients in the four sub- 
groups, we used a Student $t$ test and Chi-square test. The Statistical Package for Social Sciences, version 16, was used for data analysis. Univariate analysis was initially performed to determine predictors of lymph node status. The Kaplan-Meier method was subsequently used to estimate survival distributions for DFS and OS in participants with positive or negative lymph nodes. A log-rank test was used to assess differences between the distributions of DFS and OS, and multivariate Cox proportional hazard regression analysis was performed to assess the independent prognostic significance of clinical and histopathological variables on events or OS. Unless otherwise stated, a $\mathrm{P}<0.05$ was considered to be statistically significant.

\section{RESULTS}

\section{Patient characteristics}

A total of 3296 breast cancer patients with an age between 25 and 88 years were referred to interdisciplinary evaluation and their data was included in the institutional database from 2005 to 2007 . We subsequently included 1580 patients who were diagnosed with luminal A disease based on histopathology and immunohistochemical staining [ER and/or PgR positive, HER2-, low Ki-67 $(<14 \%)$ or grade I/II]. The analysis is therefore based on data from enrolled patients. The characteristics of assessable patients are detailed in Table 1.

\begin{tabular}{|c|c|c|c|c|c|}
\hline Parameter & All N (\%) & LN0 N (\%) & LN1 N (\%) & LN2 N (\%) & LN3 N (\%) \\
\hline Number & $1580(100)$ & $878(100)$ & $428(100)$ & $160(100)$ & $114(100)$ \\
\hline Mean age, years & $51.35 \pm 11.16$ & & & & \\
\hline \multicolumn{6}{|l|}{ Age at diagnosis (years) } \\
\hline$<35$ years & $66(4.2)$ & $41(4.7)$ & $13(3.0)$ & $6(3.75)$ & $6(5.3)$ \\
\hline$\geq 35$ and $<65$ years & $1309(82.8)$ & $705(80.3)$ & $367(85.7)$ & $140(87.5)$ & $97(85.1)$ \\
\hline$\geq 65$ years & $205(13.0)$ & $132(15.0)$ & $48(11.2)$ & $14(8.75)$ & $11(9.6)$ \\
\hline \multicolumn{6}{|l|}{ Menstrual status } \\
\hline Premenopausal & $958(60.6)$ & $526(33.2)$ & $259(60.5)$ & $104(65.0)$ & $69(60.5)$ \\
\hline Postmenopausal & $622(39.4)$ & $352(22.3)$ & $169(39.5)$ & $56(35.0)$ & $45(39.5)$ \\
\hline \multicolumn{6}{|l|}{ Complications } \\
\hline Yes & $376(23.8)$ & $213(13.5)$ & $99(23.1)$ & $33(20.6)$ & $31(27.2)$ \\
\hline No & $1204(76.2)$ & $665(42.1)$ & $329(76.9)$ & $127(79.4)$ & $83(72.8)$ \\
\hline \multicolumn{6}{|l|}{ Histologic type } \\
\hline Infiltrating ductal Carcinoma & $1475(93.4)$ & $813(51.5)$ & $413(96.5)$ & $148(92.5)$ & $101(88.6)$ \\
\hline Infiltrating lobular Carcinoma & $78(4.9)$ & $41(2.6)$ & $12(2.8)$ & $12(7.5)$ & $13(11.4)$ \\
\hline Other infiltrating Carcinoma & $27(1.7)$ & $24(1.5)$ & $3(0.7)$ & $0(0)$ & $0(0)$ \\
\hline \multicolumn{6}{|l|}{ Tumor size $(\mathrm{cm})$} \\
\hline T1 (02) & $995(63.0)$ & $613(38.8)$ & $268(62.6)$ & $75(46.9)$ & $39(34.2)$ \\
\hline T2 (2-5) & $517(32.7)$ & $233(14.7)$ & $145(33.9)$ & $74(46.3)$ & $65(57.0)$ \\
\hline $\mathrm{T} 3(>5)$ & $40(2.5)$ & $21(1.3)$ & $7(1.6)$ & $6(3.75)$ & $6(5.3)$ \\
\hline T4 (with direct extension to the chest wall and/or to the skin) & $21(1.3)$ & $8(0.5)$ & $6(1.4)$ & $5(3.1)$ & $2(2)$ \\
\hline Tx (primary tumor cannot be assessed) & $7(0.4)$ & $3(0.2)$ & $2(0.5)$ & $0(0)$ & $2(2)$ \\
\hline \multicolumn{6}{|l|}{ Vascular invasion } \\
\hline Yes & $152(9.6)$ & $42(4.7)$ & $45(10.5)$ & $29(18.1)$ & $36(31.6)$ \\
\hline No & $1428(90.4)$ & $836(95.2)$ & $383(89.5)$ & $131(81.9)$ & $78(68.4)$ \\
\hline \multicolumn{6}{|l|}{ Treatment* } \\
\hline En & $374(23.7)$ & $322(36.7)$ & $39(9.1)$ & $8(5)$ & $5(4.4)$ \\
\hline $\mathrm{En}+\mathrm{Ch}$ & $1206(76.3)$ & $556(63.3)$ & $389(90.9)$ & $152(95)$ & 109 (95.6) \\
\hline
\end{tabular}

*En = endocrine therapy; $\mathrm{Ch}=$ chemotherapy. 
The mean age of patients was 50.97 years (median: 50 years, range: $25-88$ ). Sixty-eight patients were under the age of 35 years, 1307 were between 35 and 65, and 205 were older than 65. Depending on lymph node status, patients were classified into LN0, LN1, LN2, and LN3 subgroups, and clinical outcomes were compared. The LN1, LN2, and LN3 subgroups included patients with 1-3, 4-10, and $>10$ positive nodes, respectively. There were $878(55.6 \%), 428$ (27.1\%), $160(10.1 \%)$ and 114 (7.2\%) patients, respectively, in the LN0, LN1, LN2, and LN3 subgroups. A total of 374 patients received adjuvant endocrine therapy after surgery, including $322(36.7 \%), 39(9.1 \%), 8(5 \%)$, and $5(4.4 \%)$ patients, respectively, in the LN0, LN1, LN2, and LN3 subgroups. The remainder of patients received endocrine therapy plus chemotherapy.

\section{Clinical outcomes according to lymph node status}

During a median follow-up of 67 months (range: 60-89), 184 patients experienced recurrence of disease. Of these, 123 patients experienced local recurrence, 47 had distant metastases, and 14 had both. Of the 113 patients who died within the follow-up period, 92 died of breast cancer, 5 of other malignances, and 16 of unrelated conditions (i.e., heart failure, chronic obstructive pulmonary disease, diabetes, arrhythmia, renal insufficiency). OS in all patients was $92.8 \%$, while the 5-year OS/DFS percentages for the LN0, LN1, LN2, and LN3 subgroups were 94.7/92.2, 94.2/90.0, 90.0/80.6, and 78.1/63.2\%, respectively.

At multivariate analysis, lymph node status was still significantly associated with increased risk of disease recurrence and death. Significantly lower DFS was seen in the LN2 and LN3 subgroups among tumors defined as luminal A breast cancer $(\mathrm{P}<0.001)$ compared with the LN0 subgroup. This result was confirmed at multivariate analysis $(\mathrm{HR}=2.2,95 \% \mathrm{CI}$ $=1.4-3.3 ; \mathrm{HR}=4.3,95 \% \mathrm{CI}=2.9-6.3$, respectively). Multivariate Cox regression analysis showed that the LN2 and LN3 subgroups were associated with an increased risk of overall mortality $(\mathrm{HR}=1.9,95 \% \mathrm{CI}=1.1-3.4 ; \mathrm{HR}=3.7,95 \% \mathrm{CI}=2.2-6.1$, respectively $)$ after adjustment for age, vascular invasion, and tumor size. The DFS and OS curves for the LN2 and LN3 subgroups demonstrated that increased lymph node involvement was associated with poorer prognosis for luminal A breast cancer (Table 2 and Figure 1).

The outcomes of patients in LN1 subgroup were similar to the LN0 subgroup considering DFS and OS $(\mathrm{HR}=1.3,95 \% \mathrm{CI}=0.9-1.9, \mathrm{P}=0.183$ for $\mathrm{DFS} ; \mathrm{HR}=1.2 ; 95 \% \mathrm{CI}=$ $0.7-1.9, \mathrm{P}=0.555$ for $\mathrm{OS})$.

\begin{tabular}{|c|c|c|c|c|c|c|c|}
\hline \multicolumn{2}{|c|}{ Parameter } & \multicolumn{3}{|c|}{ Disease-free survival } & \multicolumn{3}{|c|}{ Overall survival } \\
\hline & & HR & $95 \% \mathrm{CI}$ & $P$ value & HR & $95 \% \mathrm{CI}$ & $P$ value \\
\hline \multirow{4}{*}{$\mathrm{pN}$} & N0 & Ref. & - & - & Ref. & - & - \\
\hline & $\mathrm{N} 1$ & 1.3 & $0.9-1.9$ & 0.183 & 1.2 & $0.7-1.9$ & 0.555 \\
\hline & N2 & 2.2 & $1.4-3.3$ & $<0.001$ & 1.9 & $1.1-3.4$ & 0.03 \\
\hline & N3 & 4.3 & $2.9-6.3$ & $<0.001$ & 3.7 & $2.2-6.1$ & $<0.001$ \\
\hline \multirow[t]{5}{*}{ pT } & $\mathrm{T} 1$ & Ref. & - & - & Ref. & - & - \\
\hline & $\mathrm{T} 2$ & 1.4 & $1.0-1.9$ & 0.043 & 2.3 & $1.5-3.5$ & $<0.001$ \\
\hline & $\mathrm{T} 3$ & 4.2 & $2.4-7.3$ & $<0.001$ & 6.2 & $3.1-12.6$ & $<0.001$ \\
\hline & $\mathrm{T} 4$ & 2.7 & $1.2-6.1$ & 0.021 & 2.2 & $0.5-9.1$ & 0.285 \\
\hline & Tx & 3.2 & $1.0-10.3$ & 0.049 & 3 & $0.4-22.4$ & 0.276 \\
\hline Age & $\geq 65$ years & 2 & $1.4-2.9$ & $<0.001$ & 2.8 & $1.8-4.2$ & $<0.001$ \\
\hline PVI* & & 1 & $0.7-1.6$ & 0.906 & 1.1 & $0.7-1.9$ & 0.652 \\
\hline
\end{tabular}

*Peritumoral vascular invasion. 

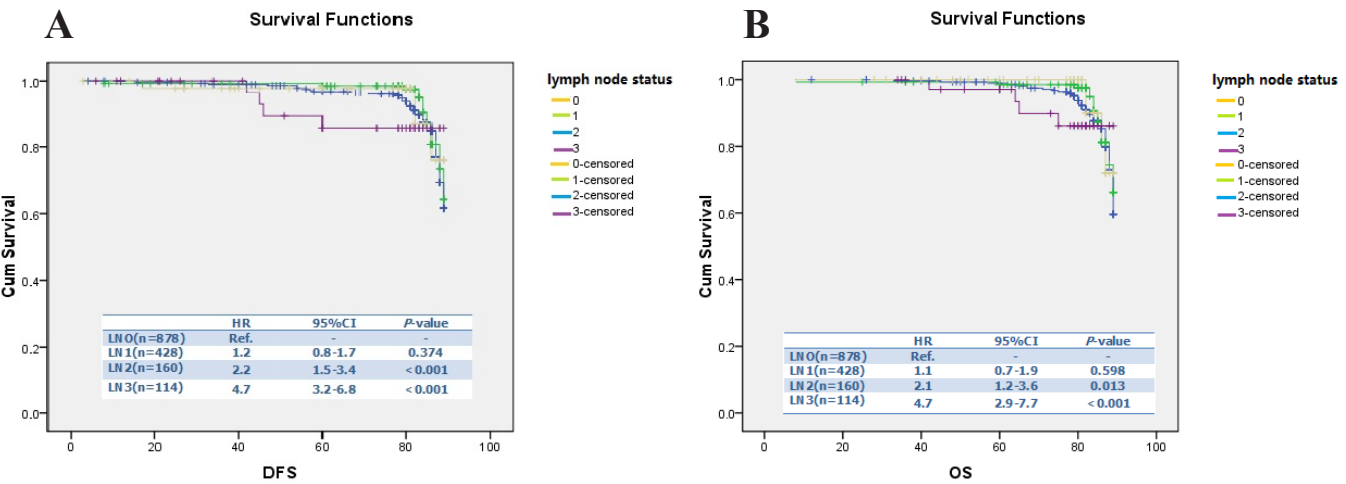

Figure 1. DFS (A) and OS (B) according to lymph node status.

\section{Adjuvant treatment}

An exploratory analysis was conducted to evaluate the impact of the different adjuvant therapies on DFS and OS in the four subgroups. As shown in Table 1, patients in the LN0 subgroup received more adjuvant endocrine therapy than the other three subgroups.

Our data showed that DFS was lower in patients in the LN0 and LN1 subgroups considering those who received chemotherapy plus endocrine therapy versus patients treated with endocrine therapy alone, although this difference was not statistically significant (Table 3, Figures 2 and 3). In addition, there was a trend towards increased $\mathrm{OS}$ ( $\mathrm{HR}=1.294$ for $\mathrm{LN0}$; $\mathrm{HR}=1.379$ for $\mathrm{LN} 1$ ).

Table 3. Clinical outcomes in all patients according to lymph node status and adjuvant treatment.

\begin{tabular}{|c|c|c|c|c|c|c|c|c|c|c|c|c|}
\hline & \multicolumn{3}{|c|}{ LN0 (322/878) } & \multicolumn{3}{|c|}{ LN1 $(39 / 389)$} & \multicolumn{3}{|c|}{ LN2 (8/152) } & \multicolumn{3}{|c|}{ LN3 (5/114) } \\
\hline & HR & $95 \% \mathrm{CI}$ & P value & HR & $95 \% \mathrm{CI}$ & P value & HR & $95 \% \mathrm{CI}$ & $P$ value & HR & $95 \% \mathrm{CI}$ & $P$ value \\
\hline \multicolumn{13}{|l|}{ DFS } \\
\hline $\mathrm{En}^{*}$ & Ref. & - & - & Ref. & - & - & Ref. & - & - & Ref. & - & - \\
\hline $\begin{array}{l}\mathrm{En}+\mathrm{Ch}^{*} \\
\text { OS }\end{array}$ & 0.847 & $0.264-2.713$ & 0.78 & 0.833 & $0.275-2.520$ & 0.746 & 0.643 & $0.081-5.087$ & 0.676 & 1.866 & $0.539-6.463$ & 0.325 \\
\hline En & Ref. & - & - & Ref. & - & - & & - & - & Ref. & - & - \\
\hline $\mathrm{En}+\mathrm{Ch}$ & 1.294 & $0.651-2.574$ & 0.462 & 1.379 & $0.368-5.228$ & 0.639 & 0.938 & $0.102-8.656$ & 0.955 & 2.08 & $0.452-9.582$ & 0.347 \\
\hline
\end{tabular}

*En = endocrine therapy; $\mathrm{CH}=$ chemotherapy.

A

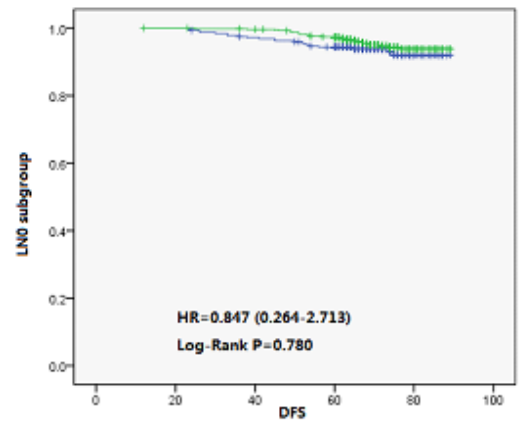

B

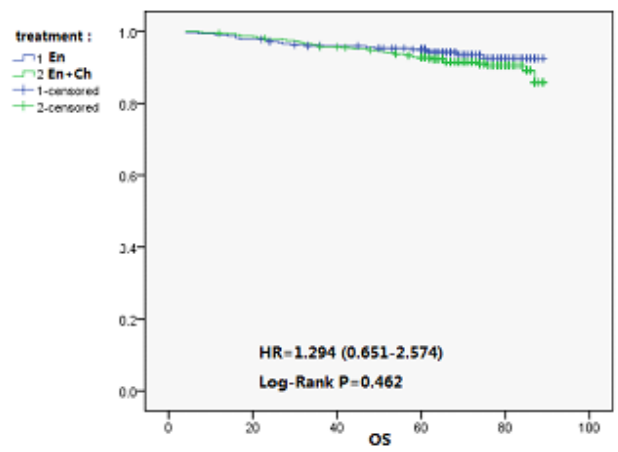

treatment : $\rightarrow-1$ - Sensoces T-2 - censored

Figure 2. DFS (A) and OS (B) according to treatment in the LN0 group. 

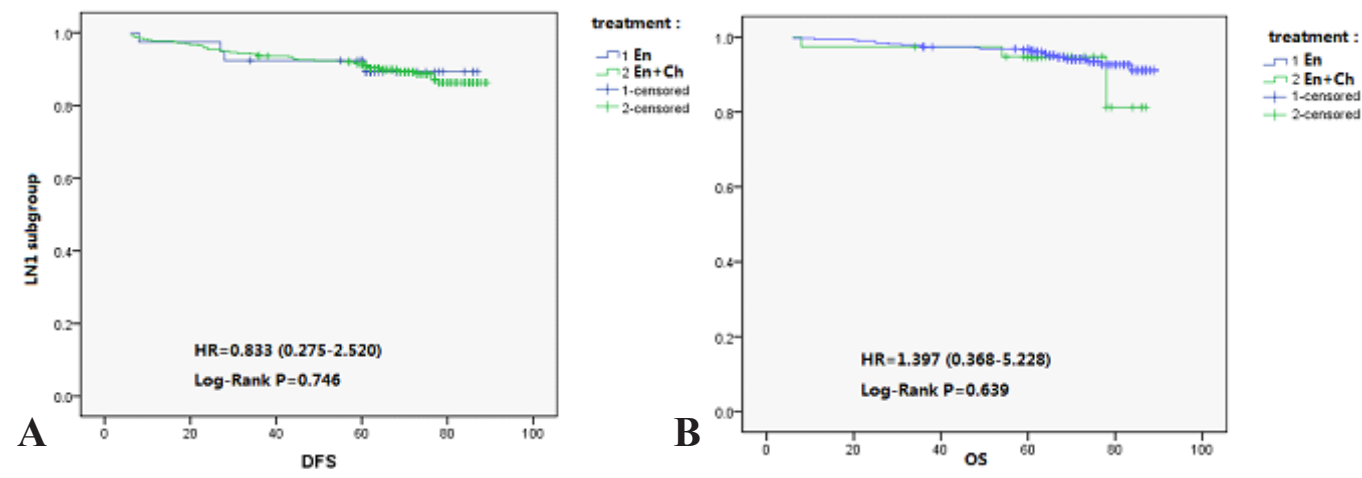

$\rightarrow 1 \mathrm{En}$
$\rightarrow 2 \mathrm{En+Ch}$
$+12 \mathrm{ens} \times \mathrm{ed}$

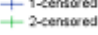

Figure 3. DFS (A) and OS (B) according to treatment in the LN1 group.

In the LN2 subgroup, there was a trend towards lower DFS and OS in patients treated with adjuvant chemotherapy plus endocrine therapy compared with patients receiving endocrine therapy alone ( $\mathrm{HR}=0.643$ for $\mathrm{DFS} ; \mathrm{HR}=0.938$ for $\mathrm{OS}$ ), although this difference was not statistically significant (Table 3 and Figure 4). However, in the LN3 subgroup, a beneficial trend on DFS and OS was not observed (Table 3 and Figure 5).
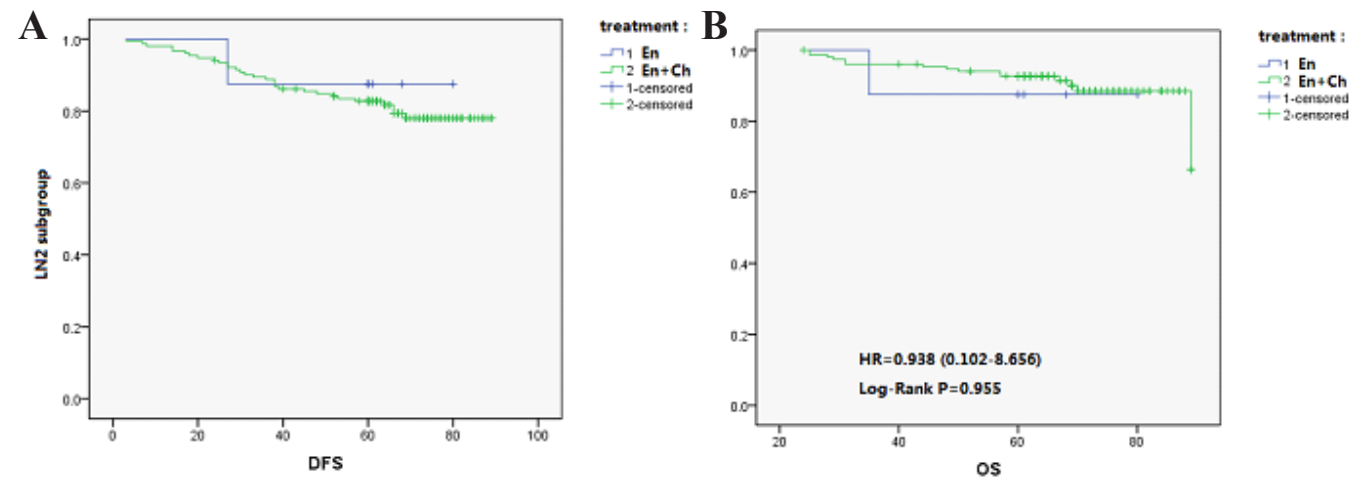

Figure 4. DFS (A) and OS (B) according to treatment in the LN2 group.

A

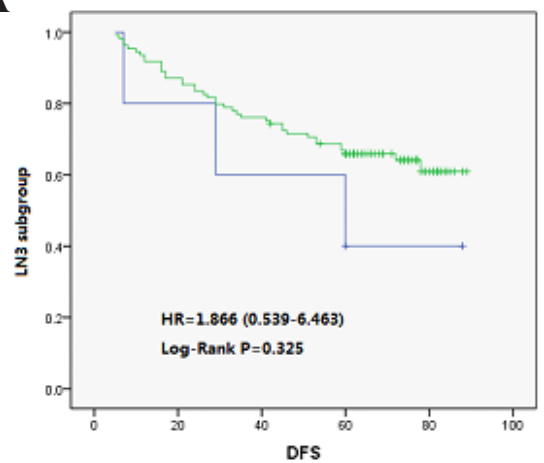

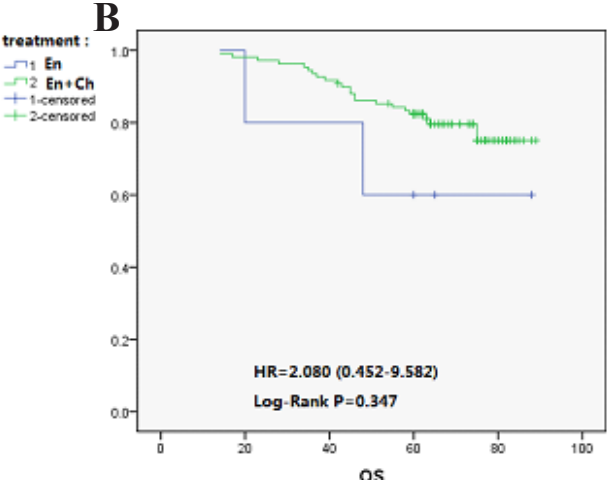

Figure 5. DFS (A) and OS (B) according to treatment in the LN3 group. 


\section{DISCUSSION}

Based on emerging knowledge in the field of tumor biology, the 12th St. Gallen International Breast Cancer Conference (2011) Expert Panel adopted a new approach to classify patients for therapeutic purposes based on the recognition of intrinsic biological subtypes within the breast cancer spectrum (Goldhirsch et al., 2011). For practical purposes, it is not always feasible to obtain information on gene expression using microarrays, and a simplified classification, closely following that proposed by Cheang et al. (2009), was adopted. Subtypes defined by clinicopathological criteria are similar, but not identical, to intrinsic subtypes and represent a convenient approximation.

Treatment strategies are tailored to the defined subtypes. For luminal A breast cancer, the Panel strongly agreed that luminal A disease was less responsive to chemotherapy, that chemotherapy was less useful in such patients, and that no preferred chemotherapy regimen could be defined for treatment of luminal A disease (Goldhirsch et al., 2011). However, it is still unclear that treatment with adjuvant chemotherapy or endocrine therapy alone for patients with luminal A breast cancer and lymph node metastasis for now. Indeed, limited information is available in the adjuvant setting on outcomes and responsiveness to therapy in the population defined as luminal A breast cancer with positive lymph nodes.

Our study showed that the involvement of $\geq 4$ axillary lymph nodes was a major predictor of metastases and mortality, with HR of 2.2 and 4.7 and 2.1 and 4.7, respectively, for the LN2 and LN3 subgroups. Results from other studies have also shown a relationship between prognosis and lymph node status. In particular, in the SEER Program of the National Cancer Institute the markedly poorer survival of women with $\geq 4$ positive axillary LNs $(21 \%$ of the present cohort) was evident for all tumor sizes. Five-year survival in patients with no positive nodes or 1-3 involved nodes was 77 and $99 \%$, respectively, compared with $64 \%$ in those with $\geq 4$ positive nodes (Carter et al., 1989).

In addition to a more aggressive disease presentation, which affects outcomes, the results of the present study led to the identification of patients who received benefit from adjuvant tailored therapies within the subgroup of patients with positive lymph nodes in luminal A breast cancer as defined by immunohistochemistry. In fact, patients in the LN2 and LN3 subgroups were at increased risk of recurrence and death compared with the LN0 subgroup. Conversely, no significant effect of lymph node status was seen in the LN1 subgroup. The results seen in terms of low number of events and similar outcomes in the LN0 subgroup might be useful to aid in developing a therapeutic algorithm for the LN1 group with regards to adjuvant treatment.

The results of this analysis are also important as they help to clarify the role of adjuvant chemotherapy in patients with luminal A breast cancer and lymph node metastasis. In particular, whether the use of adjuvant chemotherapy may be a preferred solution for these patients is a hypothesis that has not been tested adequately. Cytotoxic chemotherapy is, on average, beneficial in delaying relapse and prolonging survival in women with early stage breast cancer (EBCTCG, 2005). Therefore, this has favored its widespread use in all or nearly all women in this group. Guidelines from the 2000 National Institutes of Health Consensus Development Conference state that "because adjuvant polychemotherapy improves survival, it should be recommended to the majority of women with localized breast cancer regardless of lymph node, menopausal, or hormone receptor status" and that "at the present time, there are no convincing data to support the use of any known biologic factor in selecting a specific adjuvant chemotherapy regimen in breast cancer" (The National Institutes of Health Consensus 
Development Conference, 2001). As a result of these guidelines, most of the patients in our study received adjuvant chemotherapy after surgery. Anthracycline-containing chemotherapy, such as 4 or 6 courses of adriamycin and cyclophosphamide, was the preferred option in our patients.

Our results showed that there was a trend towards reduced DFS and OS in patients treated with adjuvant chemotherapy plus endocrine therapy compared with patients receiving endocrine therapy alone in the LN2 subgroup, although this difference was not statistically significant. However, in the LN3 subgroup, there was no benefit on either DFS or OS. There are several potential reasons that can help to explain the different responses to adjuvant chemotherapy according to lymph node status. One is that it might be related to the overall poor prognosis of the LN3 subgroup. Moreover, many patients in LN3 subgroup received 4 or 6 courses of adriamycin and cyclophosphamide. The lack of any benefit on survival in the LN3 subgroup maybe due to the insufficiency of chemotherapy regimens. Our data suggest that patients with $\geq 4$ positive lymph nodes may receive survival benefits from adjuvant chemotherapy. Moreover, paclitaxel/docetaxel-containing chemotherapy, such as 6 courses of paclitaxel/docetaxel and anthracycline or dose-dense regimens, may be beneficial for patients with luminal A breast cancer and LN3 status, although the efficacy of chemotherapy requires further study.

Our analysis showed that DFS was lower in patients with LN0 and LN1 status who received chemotherapy plus endocrine therapy compared with those treated with endocrine therapy alone, although this difference was not statistically significant. In addition, there was a trend towards increased OS. The proportion of patients dying of unrelated conditions in the LN0 and LN1 subgroups was higher than the LN2 and LN3 subgroups, similar to published studies. The IBCSG IX trial in postmenopausal women with node-negative disease found no benefit of adding cyclophosphamide, methotrexate, and fluorouracil in the prospective stratum with ER-positive disease (Aebi et al., 2011), whereas in the corresponding premenopausal study IBCSG VIII, no benefit of chemotherapy was seen among patients whose tumors had high expression of ER (Viale et al., 2008a) or low proliferation as measured by Ki-67 (Karlsson et al., 2011). Among patients with high ER expression, no case of complete pathologic remission was observed at the European Institute of Oncology (Colleoni et al., 2009). It may be argued that even if only a small proportion of patients actually receive benefit from chemotherapy, all should receive it. This ignores the potential harm of exposing a large majority of patients to the toxic, occasionally fatal, adverse effects of chemotherapy for no therapeutic advantage. The results of our study suggest that endocrine therapy is sufficient for patients with LN1 and LN0 status, and that chemotherapy should be avoided in this group of patients.

Although lymph node status remains one of the most important independent prognostic predictors for luminal A breast cancer, in those patients with 0-3 positive lymph nodes endocrine therapy is sufficient. However, patients with $\geq 4$ positive lymph nodes, and especially those with $>10$, should receive chemotherapy possibly using paclitaxel/docetaxel-containing or dose-dense regimens.

\section{Conflicts of interest statement}

The authors declare no conflict of interest.

\section{REFERENCES}

Albain KS, Barlow WE, Shak S, Hortobagyi GN, et al. (2010). Prognostic and predictive value of the 21-gene 
recurrence score assay in postmenopausal women with node-positive, oestrogen-receptor-positive breast cancer on chemotherapy: a retrospective analysis of a randomised trial. Lancet Oncol. 11: 55-65.

Aebi S, Sun Z, Braun D, Price KN, et al. (2011). Differential efficacy of three cycles of CMF followed by tamoxifen in patients with ER-positive and ER-negative tumors: long-term follow up on IBCSG Trial IX. Ann. Oncol. 22: 1981-1987.

Blows FM, Driver KE, Schmidt MK, Broeks A, et al. (2010). Subtyping of breast cancer by immunohistochemistry to investigate a relationship between subtype and short and long term survival: a collaborative analysis of data for 10,159 cases from 12 studies. PLoS Med. 7: e1000279.

Carter CL, Allen C and Henson DE (1989). Relation of tumor size, lymph node status, and survival in 24740 breast cancer cases. Cancer 63: 181-187.

Cheang MC, Chia SK, Voduc D, Gao D, et al. (2009). Ki67 index, HER2 status, and prognosis of patients with luminal B breast cancer. J. Natl. Cancer Inst. 101: 736-750.

Colleoni M, Bagnardi V, Rotmensz N, Gelber RD, et al. (2009). Increasing steroid hormone receptors expression defines breast cancer subtypes non responsive to preoperative chemotherapy. Breast Cancer Res. Treat. 116: 359-369.

Colleoni M, Cole BF, Viale G, Regan MM, et al. (2010). Classical cyclophosphamide, methotrexate, and fluorouracil chemotherapy is more effective in triple-negative, node-negative breast cancer: results from two randomized trials of adjuvant chemoendocrine therapy for node-negative breast cancer. J. Clin. Oncol. 28: 2966-2973.

Di Cosimo S and Baselga J (2010). Management of breast cancer with targeted agents: importance of heterogenicity. Nat. Rev. Clin. Oncol. 7: 139-147.

Dignam JJ, Dukic V, Anderson SJ, Mamounas EP, et al. (2009). Hazard of recurrence and adjuvant treatment effects over time in lymph node-negative breast cancer. Breast Cancer Res. Treat. 116: 595-602.

Early Breast Cancer Trialists' Collaborative Group (EBCTCG) (2005). Effects of chemotherapy and hormonal therapy for early breast cancer on recurrence and 15-year survival: an overview of the randomised trials. Lancet 365: 1687-1717.

Elston CW and Ellis IO (1991). Pathological prognostic factors in breast cancer. I. The value of histological grade in breast cancer: experience from a large study with long-term follow-up. Histopathology 19: 403-410.

Goldhirsch A, Wood WC, Senn HJ, Glick JH, et al. (1995). Meeting highlights: international consensus panel on the treatment of primary breast cancer. J. Natl. Cancer Inst. 87: 1441-1445.

Goldhirsch A, Ingle JN, Gelber RD, Coates AS, et al. (2009). Thresholds for therapies: highlights of the St Gallen International Expert Consensus on the primary therapy of early breast cancer 2009. Ann. Oncol. 20: 1319-1329.

Goldhirsch A, Wood W, Coates A, Gelber RD, et al. (2011). Strategies for subtypes - dealing with the diversity of breast cancer: highlights of the St Gallen International Expert Consensus on the Primary Therapy of Early Breast Cancer 2011. Ann. Oncol. 22: 1736-1747.

Hugh J, Hanson J, Cheang MCU, Nielsen TO, et al. (2009). Breast cancer subtypes and response to docetaxel in node-positive breast cancer: use of an immunohistochemical definition in the BCIRG 001 trial. J. Clin. Oncol. 27: 1168-1176.

Karlsson P, Sun Z, Braun D, Price KN, et al. (2011). Long-term results of International Breast Cancer Study Group Trial VIII: adjuvant chemotherapy plus goserelin compared with either therapy alone for premenopausal patients with node-negative breast cancer. Ann. Oncol. 22: 2216-2226.

Liedtke C, Mazouni C, Hess KR, André F, et al. (2008). Response to neoadjuvant therapy and long-term survival in patients with triple-negative breast cancer. J. Clin. Oncol. 26: 1275-1281.

Millikan RC, Newman B, Tse C-K, Moorman PG, et al. (2008). Epidemiology of basal-like breast cancer. Breast Cancer Res. Treat. 109: 123-139.

Nguyen PL, Taghian AG, Katz MS, Niemierko A, et al. (2008). Breast cancer subtype approximated by estrogen receptor, progesterone receptor, and HER-2 is associated with local and distant recurrence after breast-conserving therapy. $J$. Clin. Oncol. 26: 2373-2378.

Nielsen TO, Hsu FD, Jensen K, Cheang M, et al. (2004). Immunohistochemical and clinical characterization of the basallike subtype of invasive breast carcinoma. Clin. Cancer Res. 10: 5367-5374.

Paik S, Tang G, Shak S, Kim C, et al. (2006). Gene expression and benefit of chemotherapy in women with node-negative, estrogen receptor-positive breast cancer. J. Clin. Oncol. 24: 3726-3734.

Phipps AI, Chlebowski RT, Prentice R, McTiernan A, et al. (2011a). Body size, physical activity, and risk of triplenegative and estrogen receptor-positive breast cancer. Cancer Epidemiol. Biomarkers Prev. 20: 454-463.

Phipps AI, Buist DS, Malone KE, Barlow WE, et al. (2011b). Reproductive history and risk of three breast cancer subtypes defined by three biomarkers. Cancer Causes Control 22: 399-405.

Rosen PP and Oberman HA (1993). Tumors of the mammary gland. Armed Forces Institute of Pathology, American Registry of Pathology, Washington.

Sotiriou C, Neo S-Y, McShane LM, Korn EL, et al. (2003). Breast cancer classification and prognosis based on gene expression profiles from a population-based study. Proc. Natl. Acad. Sci. U. S. A. 100: 10393-10398.

Tang G, Shak S, Paik S, Anderson SJ, et al. (2011). Comparison of the prognostic and predictive utilities of the 21- 
gene Recurrence Score assay and Adjuvant! for women with node-negative, ER-positive breast cancer: results from NSABP B-14 and NSABP B-20. Breast Cancer Res. Treat. 127: 133-142.

The National Institutes of Health Consensus Development Conference (2001). Adjuvant therapy for breast cancerBethesda, Maryland, USE: November 1-3, 2000. Proceedings. J. Natl. Cancer Inst. Monogr. 30: 1-152.

van't Veer LJ, Dai H, Van De Vijver MJ, He YD, et al. (2002). Gene expression profiling predicts clinical outcome of breast cancer. Nature 415: 530-536.

Viale G, Bosari S, Mazzarol G, Galimberti V, et al. (1999). Intraoperative examination of axillary sentinel lymph nodes in breast carcinoma patients. Cancer 85: 2433-2438.

Viale G, Regan MM, Marorano E, Galimberti V, et al. (2008a). Chemoendocrine compared with endocrine adjuvant therapies for node-negative breast cancer: Predictive value of centrally reviewed expression of estrogen and progesterone receptors-International Breast Cancer Study Group. J. Clin. Oncol. 26: 1404-1410.

Viale G, Regan MM, Mastropasqua MG, Maffini F, et al. (2008b). Predictive value of tumor Ki-67 expression in two randomized trials of adjuvant chemoendocrine therapy for node-negative breast cancer. J. Natl. Cancer Inst. 100: 207-212.

Wo JY, Taghian AG, Nguyen PL, Raad RA, et al. (2010). The association between biological subtype and isolated regional nodal failure after breast-conserving therapy. Int. J. Radiat. Oncol. Biol. Phys. 77: 188-196. 